\title{
Fat Augmentation as Adjunct to CORE Facelift Surgery
}

\author{
Capi C. Wever, MD, $\mathrm{PhD}^{1}$ \\ ${ }^{1}$ Department of Otolaryngology, Head \& Neck Surgery, Leiden \\ University Medical Center, Albinusdreef, Leiden, The Netherlands \\ Facial Plast Surg 2021;37:224-232.
}

\begin{abstract}
Address for correspondence Capi C. Wever, MD, PhD, Wever Clinic, Benoordenhoutseweg 227-b, 2596 BE, The Hague, The Netherlands (e-mail: capi.wever@xs4all.nl).
\end{abstract}

\begin{abstract}
Ruling out cases with strong jawlines, well-developed mid-cheek regions, and good fat presence, if done artistically, fat augmentation strongly assists midface definition and can replenish hollowed regions of the face. This is particularly true for candidates with low bodyfat or smaller skull structures. Hence, fat grafting is a strong adjunct to

Keywords

- facelift

- lipofilling

- fat transfer

- surgery
\end{abstract}

Fat grafting the face has been around for over 120 years, though initially it was primarily for the purpose of correcting facial defects. ${ }^{1-4}$ Yet it has only been for approximately 20 to 30 years that the technique has found its way into the limelight of rejuvenation surgery, facilitated by the arrival of liposuction to the toolbox of plastic surgeons in the 1980s. ${ }^{5}$ As the technique became more widespread many facelift surgeons began to add fat injections to traditional excisional techniques. ${ }^{6,7}$ Yet the introduction of fat grafting has, at times, been somewhat controversial. This occurs particularly compared with the golden standard of facelift surgery, which today can be summarized as a comprehensive release of retaining ligaments in the face and excision of fat, muscle, and glands in the neck (CORE). Several factors contribute to this. Simple business logic is one of them. Injecting fat prior to or during facelift surgery adds operating time and hence adds costs to the procedure. The graft take debate makes this even more relevant, as potential resorption implies that cases may return for touch-ups. Third, negative results of overcorrection have associated fat injections to the kind of results that many surgeons do not want to identify with. Finally, the professional debate over the merits of facelift surgery, where some maintain that rejuvenating the midface is basically completely efficacious through CORE facelift techniques alone. In this review we will address all of these issues. We will conclude that fat augmentation is a valuable tool indeed, regardless of facelift technique, experience, or embedded geographical location.

\section{The Aging Process and Volume Loss}

Aging occurs at all levels of facial anatomy. This includes, but is not restricted to, ligamentous slacking and prolapse of soft tissues along a vertical oblique line, the line that we aim to lift in traditional facelift surgery. Bone and fat resorption certainly also play a role and occur depending on the variety of factors. Of course, genetic predisposition plays a role, but so does lifestyle and changes in body weight. Volume loss implies that the skin soft tissue envelope is inadequately supported, which likely facilitates many of the aging phenomena. Rohrich has stated that "morphologic changes in bony structures affect soft-tissue position...." ${ }^{8}$ However, it may also lead to muscle hyperactivity and hence the kind of rhytids that we commonly treat with botulin toxin.

Sequential computed tomography studies have shown that changes occur to the skull as we age, mostly affecting the orbit and midface, with an onset of around the third decade. ${ }^{9-12}$ Though changes vary individually, we generally see a widening of the bony orbital aperture. ${ }^{11}$ This occurs most strongly superior-medially, which is commonly referred to as A-frame published online February 3, 2021
Issue Theme Facial Rejuvenation: A Multidimensional and Multimodal Perspective; Guest Editor: Fred G. Fedok, MD, FACS (c) 2021. Thieme. All rights reserved. Thieme Medical Publishers, Inc., 333 Seventh Avenue, 18th Floor, New York, NY 10001, USA
DOI https://doi.org/ 10.1055/s-0041-1722893. ISSN 0736-6825. 
deformity. Second, we tend to see it occurring inferior-lateral, arguably giving rise to crows-feet deformity and aggravating lateral hooding. Depression of the orbital floor occurs by approximately 1 to $2 \mathrm{~mm}$, and inward rotation of the inferior orbital rim and retrusion of the midface-maxilla have been reported. ${ }^{13,14}$ Ptosis of the superficial malar fat pad, deepening of the nasolabial fold, and lengthening of the lid-cheek junction may be related to these changes. In the lower face, the mandible body height and length decrease, lowering chin volume and projection, giving rise to the deepening of the prejowl sulcus. ${ }^{11,15}$ This may also affect the tension of the platysma and hence provoke aging of the neck, in addition to cervical shortening which occurs especially in osteoporosis. All of these changes imply a smaller skull structure to support the facial tissues.

Aside from becoming ptotic, fat also tends to become atrophic as we age. Shaw et al believes this is the greatest contributor to volume loss. ${ }^{11}$ It has been suggested that the superficial fat pads are more susceptible to ptosis, and hence ought to be approached by lifting techniques, while the deep fat pads mostly deflate. At the level of the forehead, we see involution of the deep galeal fat pads, including the brow fat pad, leading to a bony and hard appearance in some, and coexplaining the deepening of glabellar and frontal rhytids. ${ }^{16}$ Preaponeurotic orbital fat tends to atrophy, provoking dermatochalasis or, alternatively, a hollowed look. The lid-cheek junction becomes elongated, and the tear trough deepens. ${ }^{16}$ The temporal fat pad also involutes, giving rise to temporal wasting. Lips generally become thinner, and the buccal hollows are also a common region for atrophy. The malar fat pad appears to deflate, as well as sag into an inferior oblique direction.

Hence there is clear pathophysiological argument to augmenting volume for rejuvenation, especially in the periorbital and (deep) zygomatic-malar region. A separate argument for the implementation of fat injections in the aging face protocol, is the potential benefit of fat to skin quality. More or less coincidentally and anecdotally, it was found that when fat was injected into the face, especially in the subdermal layers, some cases who exhibited sun damage or scarring, improved significantly in terms of skin texture and quality. ${ }^{16,17}$ Zuk was the first to describe adipose-derived multipotent stem cells, residing in the perivascular stroma of fat, later described by Moseley as adipose-derived stem-cells (ASCs). ${ }^{18,19}$ Adding ASCs to fat grafting, so called cell-assisted lipo-transfer has been suggested to improve graft retention in several studies, yet the safety and viability in facial plastic surgery is uncertain ${ }^{4}$ (-Figs. 1).

\section{Logistics of the Procedure}

Fat injections are performed at the beginning of facelift surgery. Initiating a facelift procedure with fat injections is a personal preference, though some arguments apply. The lack of swelling at the onset of the procedure is the most important argument to proceed in this fashion. Also limiting the time between harvesting and injecting may benefit the survival of fat grafts. ${ }^{20}$ Entry points and areas to be injected are marked onto the face preoperatively in the sitting position. The donor region and face are prepped, but we use limited drapes at this stage, and the surgeon only wears sterile gloves but no gown. Prepping is done with povidoneiodine solution. The hair is left undraped, yet is shampoo prepped with betadine. Before prepping and draping, local anesthesia is applied. Lidocaine $2 \%$ plus epinephrin $1: 100.000$ is used for regional nerve blocks to V1, V2, V3 as well as to the planned entry sites for harvesting and injecting. The face is not otherwise infiltrated in the direct area of planned lipofilling, hence, to avoid swelling which obscures visual feedback.

Grafting the face is about transferring micro aliquots of fat, not more than 0.02 to $0.03 \mathrm{~mL}$ per pass. So, using harvester cannulas that are small enough to harvest lipocytes that can be injected through 0.9 to $1.2 \mathrm{~mm}$ blunt cannulas is essential. For harvesting we prefer $2.4 \mathrm{~mm} \times 20 \mathrm{~cm}$ Tonnard harvester (Tulip Medical). In EU, disposable harvesting material is becoming standard, yet we have experienced that these products can lack reliability. Harvesting initiates with 11 knife stab incisions, after which a local injector is used to infiltrate the area with approximately 10 to $15 \mathrm{~mL}$ of $0.1 \%$ lidocaine plus $1: 500.000$ epinephrine. ${ }^{16}$ Hand vacuum up to a maximum of $5-\mathrm{mL}$ negative pressure in a $10-\mathrm{mL}$ syringe is used.

We harvest 1.5 times the amount that is estimated to be required. The primary harvest location is the inferior abdomen, which usually is readily available for significant harvesting, even in low body mass index (BMI) cases. If not available, due to previous abdominoplasty, for example, we retreat to the lateral and inner thigh subsequently. There is no conclusive evidence which suggests that one donor site has better fat

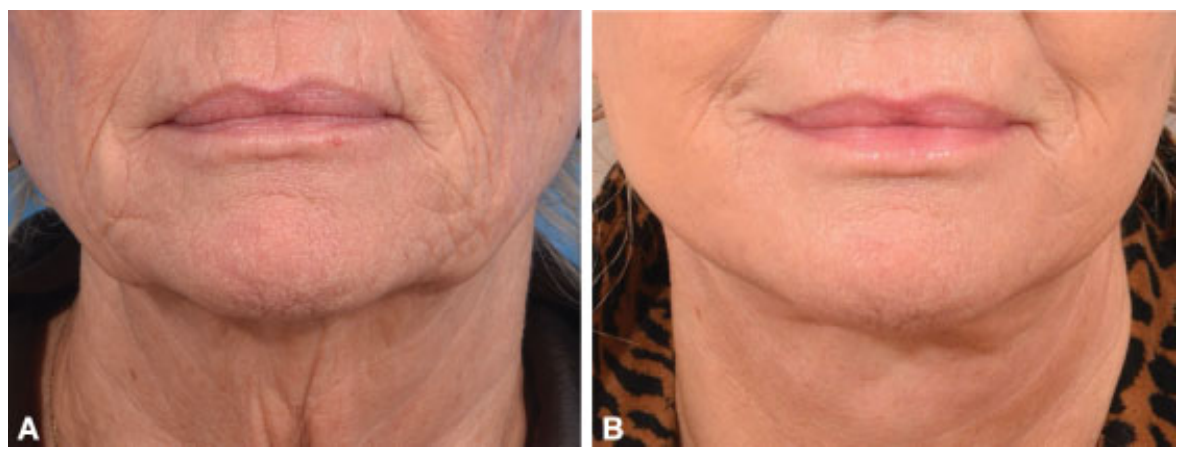

Fig. 1 (A,B) A 62-year-old, with improved skin texture after CORE facelift $+30 \mathrm{~mL}$ fat augmentation. 
quality over another. ${ }^{16}$ In most but those with the lowest BMI or those who have the history of abdominoplasty, the first two regions are usually sufficient to harvest 15 to $45 \mathrm{~mL}$ of injectable fat.

Discussions on processing techniques are abundant, yet generally more about art than science. There are many ways to do this, ranging from washing to using a centrifuge. In general, it does not seem to matter significantly how fat is prepared. ${ }^{4,7,20}$ Commercial pre-prep products have no benefit over conventional methods. We use a centrifuge at $2,000 \mathrm{rpm}$ for 2 minutes. We first remove the infranatant bottom which consists of lidocaine and other fluids. Next the supernatant oily top is removed. A transfer hub is used to transfer fat to multiple $1 \mathrm{~mL}$ syringes. Allen and Heitland suggest that the higher concentration portions of fat are those most effective in terms of the concentration of progenitor cells and anticipating the stem-cell effect. ${ }^{17,21}$

For fat injections we use multiple 1-mL Luer lock syringes. For most recipient sites the $0.9 \mathrm{~mm} \times 5 \mathrm{~cm}$ cannula is the preferred tool (Tulip Medical). For the temporal fossa, 1.2-mm canula is used to avoid accidental puncturing of perforating veins. In terms of injection depth, the deep subcutaneous and deep supraperiosteal planes are preferred. Superficial injections have the risk of visible fat lobules, yet are powerful in terms of augmentation.

We adhere to the injection technique advocated by Marten, who compares fat injections to an airbrush technique, using 20 to 30 passes per milliliter injected. This avoids congregating fat at a single location and optimizes vascular supply. ${ }^{22}$ The single hand technique, where the plumber is placed in the palm of the injecting hand, is convenient. ${ }^{20,22}$ The average injection schedule is depicted below and compared with the schedule advocated by Marten ${ }^{20,22}$ (-Table 1).

Table 1 Injection schedule versus Marten

\begin{tabular}{|l|l|l|l|}
\hline Area & Wever $(\mathrm{mL})$ & Marten $(\mathrm{mL})$ & $\begin{array}{l}\text { Injection } \\
\text { level }\end{array}$ \\
\hline Lips & $0-3$ per lip & $0-4$ per lip & Superficial \\
\hline $\begin{array}{l}\text { Temporal } \\
\text { fossa }\end{array}$ & $0-4$ per side & $0-5$ per side & Superficial \\
\hline Radix & $0-2$ & - & Deep \\
\hline Frontal & $0-3$ per side & - & Superficial \\
\hline Cheek & $0-4$ per side & $0-7$ per side & Mixed \\
\hline $\begin{array}{l}\text { Inferior } \\
\text { orbit }\end{array}$ & $0-2$ per side & $0-4$ per side & Deep \\
\hline $\begin{array}{l}\text { Superior } \\
\text { orbit }\end{array}$ & $0-2$ per side & $0-3$ per side & Deep \\
\hline $\begin{array}{l}\text { Buccal } \\
\text { hollow }\end{array}$ & $0-3$ per side & - & Superficial \\
\hline Nasolabial & $0-2$ per side & $0-3$ per side & Mixed \\
\hline Labiomental & $0-1$ per side & $0-3$ per side & Superficial \\
\hline Chin & $0-4$ & $0-6$ & Deep \\
\hline Prejowl & $0-2$ per side & 2 per side & Deep \\
\hline $\begin{array}{l}\text { Mental } \\
\text { crease }\end{array}$ & $0-2$ & - & Superficial \\
\hline
\end{tabular}

Compared with Marten and others, our schedule is on the low side. This may suggest an attempt to err on the conservative side. To err on the overcorrection side is a more serious problem, as it cannot be easily undone. On this note it is essential to explicitly ask for weight fluctuations. ${ }^{7}$ If fat is augmented it will fluctuate upward if weight is gained. Identically, augmenting fat in the process of weight loss has risks as the long-term maintenance of weight has not been established yet.

Generally, it is assumed that around 30 to $40 \%$ of autologous fat resorbs, usually within the first few months after the procedure. ${ }^{16}$ Although this is less than Peer's historic report of $50 \%$ not surviving, it still is significant and one reason why some surgeons are hesitant to adopt fat injections into their practice. ${ }^{23,24}$ Location of injection is believed to be a variable in terms of graft retention, with areas such as lips and nasolabial folds performing the poorest.

\section{Results}

If volume is not a significant issue, there is obviously no need for fat augmentation (-Fig. 2). This woman in her early 40s complained mostly of her neck and jawline. Volume was added to compensate her micrognathia with a conventional anatomical silastic chin implant (Implantech). A short-scar CORE facelift was sufficient to deal with her ptotic midface.

This very fit runner complained mostly about her hollowed appearance and perioral skin redundancy and rhytids. After a series of filler treatments, it was decided to offer her a short-scar CORE facelift with fat added to her midface and buccal hollows (-Fig. 3 ).

This 52-year-old woman complained of her tired look. Based on her youth pictures she clearly was fat depleted on top of a weakly developed midface (Jacono class III). She had undergone transcutaneous lower lid surgery years earlier elsewhere, perhaps exacerbating her problems. A short-scar CORE facelift was performed, and $25 \mathrm{~mL}$ of fat was added, of which the majority to her mid and lateral cheek region (-Fig. 4 ).

This 58-year-old complained of both sagging of her neck and jawline as well as aging of her sun-damaged skin, with superficial rhytids across much of her cheeks and buccal region. We did a full-scar CORE facelift, as well as an endoscopic forehead lift. $22 \mathrm{~mL}$ of fat was added, of which approximately $3 \mathrm{~mL}$ (per side) was superficially placed in the buccal region (-Figs. $\mathbf{5}$ ).

\section{Risks and Complications}

Complications in fat grafting are relatively rare and usually pertain to a suboptimal aesthetic outcome. Under or overcorrection are the most well-known, and should perhaps not be categorized as a complication in the truest sense. Serious complications that we need to consider are infection and vascular embolization.

\section{Prolonged Edema}

Postoperative edema typically lasts longer if facelift surgery is combined with fat augmentation, caused by the repetitive 

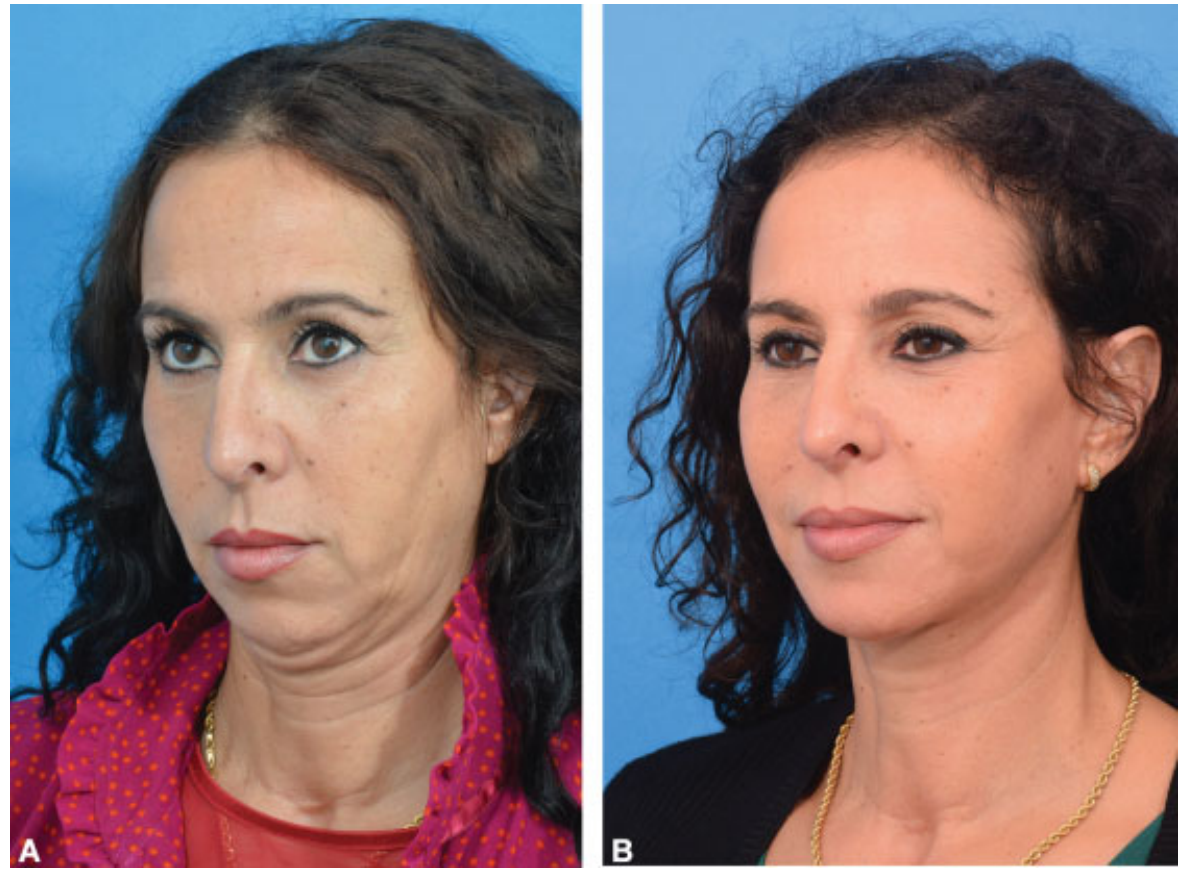

Fig. 2 (A,B) A 42-year-old, with short-scar CORE facelift + chin implant, no fat added.
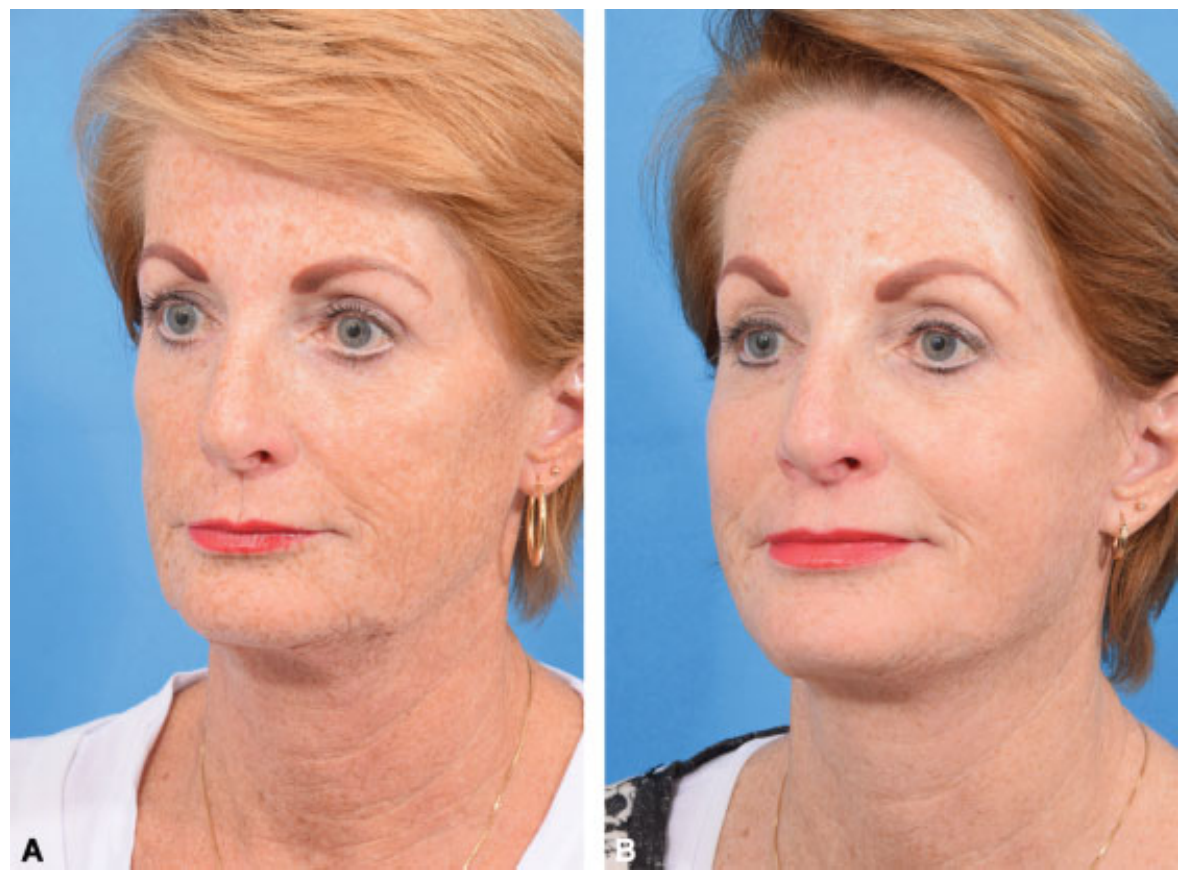

Fig. 3 (A,B) A 64-year-old, with CORE facelift $+24 \mathrm{~mL}$ fat transfer.

canula movement through soft tissue. ${ }^{20}$ Usually this is not a negative per se, as mild edema is commonly experienced favorably. But in selected cases edema can be excessive and can last from many weeks to months, leading to a need for reinforcement and support. This occurs most strongly in the periorbital region, especially if injections were extended to the lower orbital ridge and particularly if festoons and malar mounds were pre-existent. Fat grafting can exacerbate these phenomena and last for many months, aggravating the patients.

\section{Overcorrection}

Like in any other surgical procedure, risks occur mostly at the ceiling level. The best defense hence may be to under correct initially and slowly expand volume and more complex maneuvers as experience grows. Overcorrection is difficult to undo, and wrongly placed fat can be very difficult to deal with. Under correcting also has consequences for the practice, however, after edema subsides, it can lead to repeat requests for more fat, hence yielding new costs which are potentially a burden. 

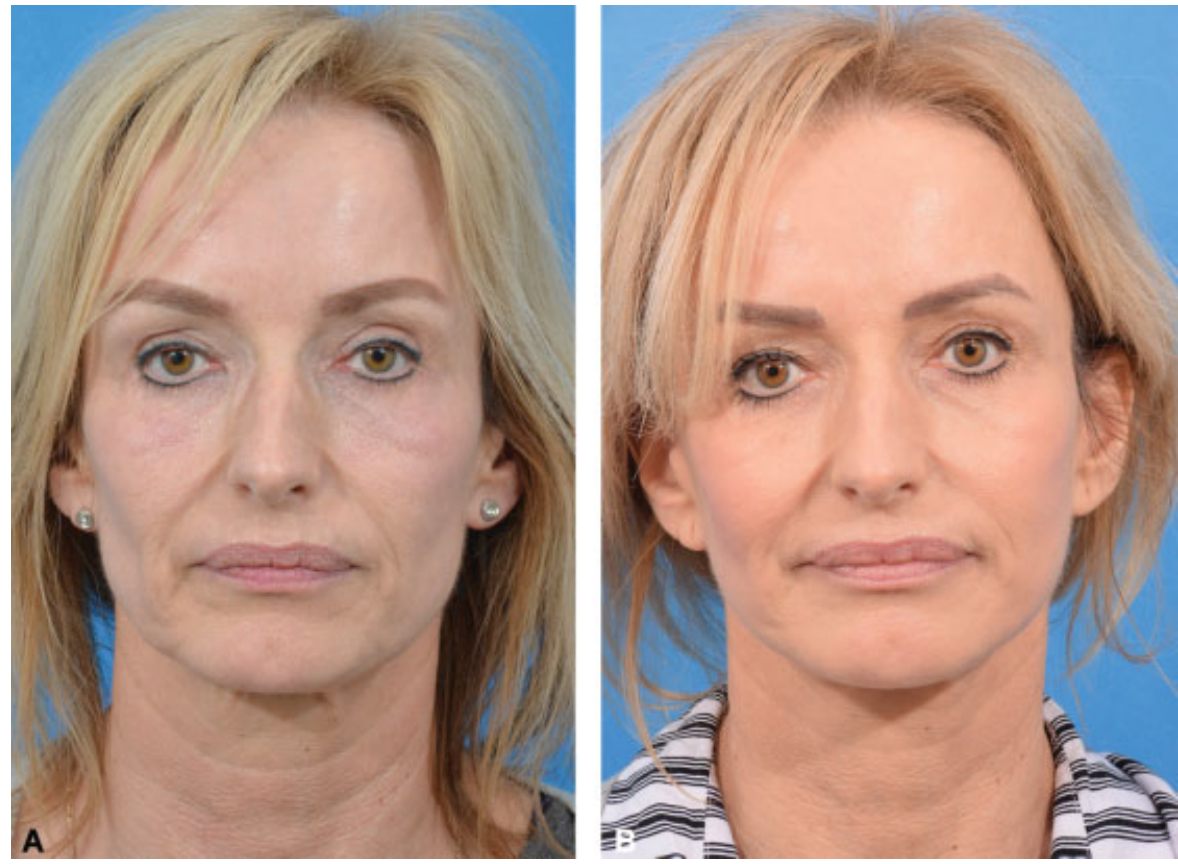

Fig. 4 (A,B) A 52-year-old with CORE facelift $+25 \mathrm{~mL}$ fat transfer.
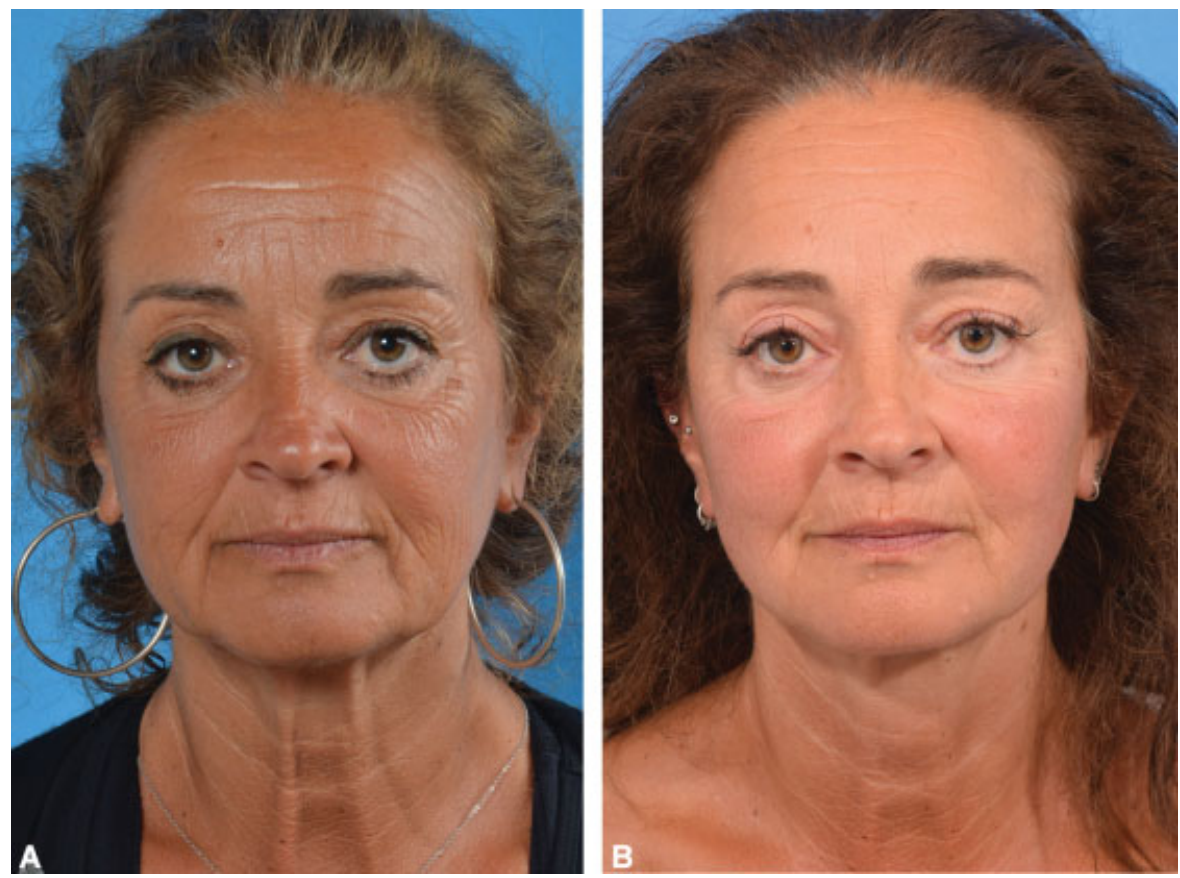

Fig. 5 (A,B) A 58-year-old CORE facelift $+22 \mathrm{~mL}$ fat transfer + endoscopic forehead lift.

\section{Irregularities and Lumps}

Contour irregularities are some of the most common complications in fat grafting, especially if injecting in a superficial plane. Of all the facial regions, the lower orbit is the most infamous. ${ }^{24-26}$ The correct plane is supraperiosteal, deep to the orbicularis oculi muscle. If fat is injected into the orbicularis oculi, the high blood supply in that area can actually provoke growth and worsen visibility. If minor irregularities occur, treatment with biweekly triamcinolone
(5 or $10 \mathrm{mg} / \mathrm{mL}$ ) or off-label 5 -flouraucil $50 \mathrm{mg} / \mathrm{mL}$ injections may help to mitigate the problem. ${ }^{7,24,27}$ If more severe, surgical evacuation through a transconjunctival or transcutaneous approach is the standard of care. Bolus injection "lumps" can be avoided by resorting to small bore 0.7 to $1.2-\mathrm{mm}$ cannulas and using multiple passes to inject only small alloquads of fat. If the plumber resists, the cannula is removed and replaced rather than putting more force on the plumber (-Fig. $\mathbf{6}$ ). 


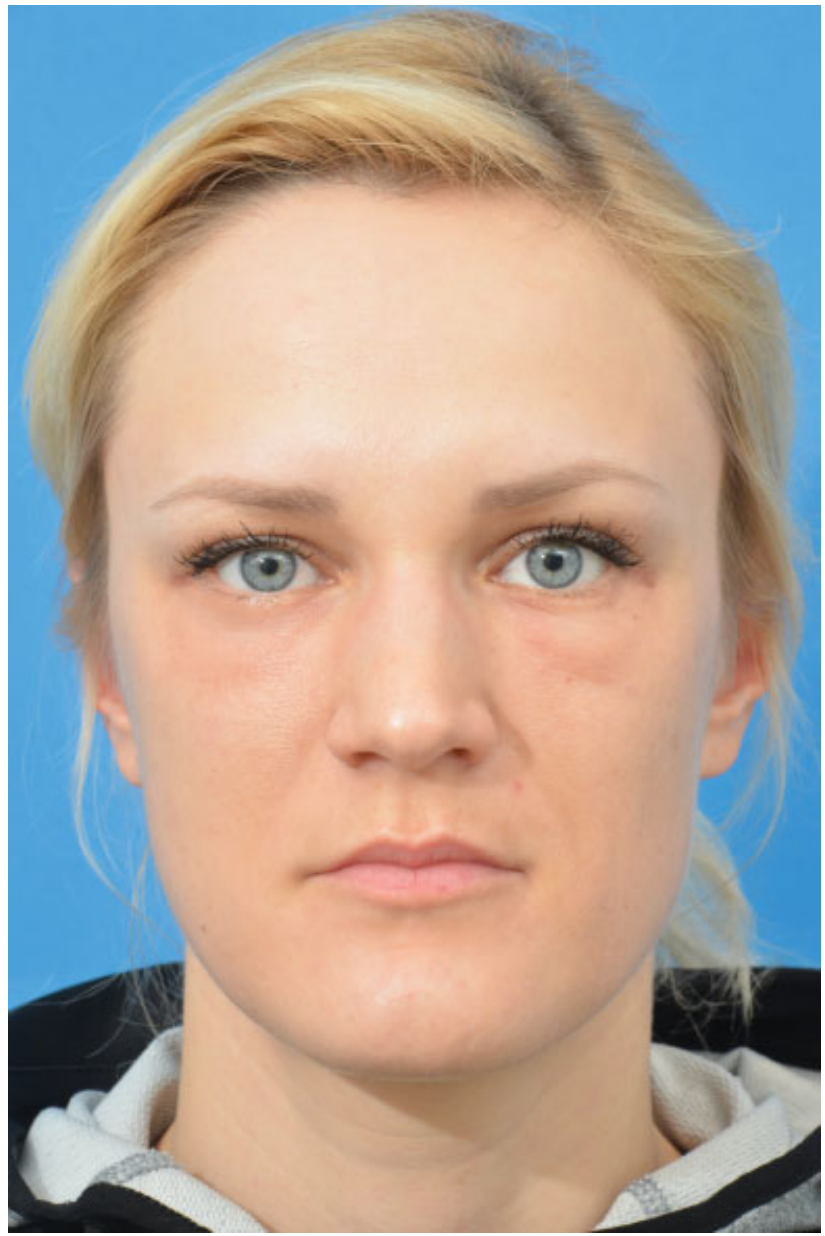

Fig. 6 Superficially placed periorbital injection of fat.

\section{Oil Cyst and Calcification}

Accidental bolus injections of fat can lead to visible or palpable lumps, which can be hard to deal with. Inadequate blood supply to the central portion can lead to necrosis of adipocytes and cicatrization, and consequently a permanent lump. ${ }^{28}$ If the necrotic area is large enough ( $>10 \mathrm{~mm}$ ), oil cysts can develop over the course of a year. Given the low volumes that are used on the face, these cysts have mostly been reported in fat grafting to the breast and buttocks. Oil cysts have a tendency to be permanent and often require surgical removal. ${ }^{29,30}$

\section{Infection}

Though rare, infection can occur. Perioperative antibiotics are a routine for many surgeons. For the author that regimen includes $1 \mathrm{~g}$ of cefazolin 30 minutes before the procedure, and a weeklong oral regimen of amoxicillin/clavulanic acid. Contamination can occur during the injection process, most likely caused by skin microorganisms. Treating the lips last can help to reduce this risk. Worse is the risk of contamination related to instrumentation, usually by Mycobacterium abscessus, which typically presents itself in a delayed fashion (6-90 days after treatment, mostly within 2 weeks) and can have a dramatic course. ${ }^{24}$ In the series $(n=12)$ described by Chen, fever was the presenting symptom, followed by granulomatous skin abscesses and tissue

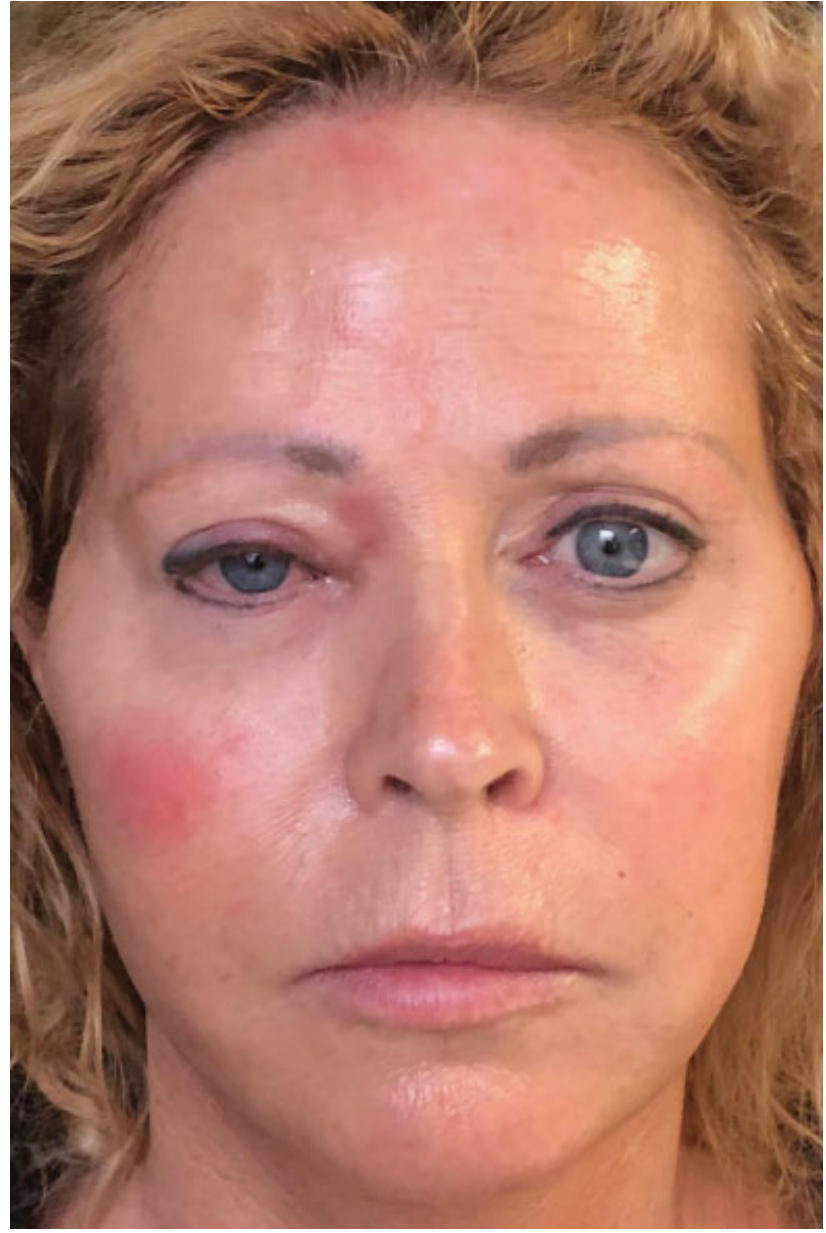

Fig. 7 Herpes ophthalmicus after revision facelift + fat transfer at 4 weeks postoperatively.

loss. $^{31,32}$ The diagnosis is commonly initially overlooked. Cultures and biopsies confirm the diagnosis. Mycobacteria infection has been related to inadequate (nonsterile) waterbased cleaning practices and has mostly been reported from Asia. Small cannulas can be difficult to clean effectively, which can induce a porous inner wall creating a favorable environment for microfilm where mycobacteria can settle. ${ }^{32}$ We consequently prefer to use single-use instrumentation in spite of premium costs and sometimes moderate durability. A 6 to 12 months macrolide-(mostly clarithromycin) or rifampinbased regimen, sometimes combined with ciprofloxacin or other antibiotics, and surgery is the mainstay of most treatment algorithms. Herpes zoster is another infection that can occur. All patients with a history of facial herpes are pretreated with valacyclovir. Yet even with a negative history, herpetic infections can occur (-Fig. 7).

\section{Nerve Injury}

If blunt cannulas are used, permanent nerve injury is rare but has been reported. Similar to facelift surgery, damage is most commonly reported to the frontal and marginal branches.

\section{Vascular Complications}

Vision loss or stroke has been described in periorbital fat injections, more or less through a similar occlusion pathway 
as in injectable fillers. ${ }^{28,33}$ It is assumed that retrograde embolization from the dorsal nasal artery, supraorbital artery, or supratrochlear artery can occur, which can forcibly backflow into the central retinal artery (Zinn's artery), hence occurring mostly on injecting the glabella or nasal dorsum. ${ }^{34}$ Yet this mechanism has been described in other locations as well, such as the nasolabial region (13\% of cases). Indeed, anastomosis between the angular artery and the dorsal nasal, supratrochlear and supraorbital arteries has been described. ${ }^{35}$

Irreversible blindness occurs within 3 hours. Many cases were accompanied by stroke, some also by skin necrosis. Zinn's artery is a branch of the ophthalmic, which in turn is a branch of the internal carotid artery, hence explaining that stroke can occur if the internal carotid artery is reached. Although experience with filler cases suggests that high-volume injections often underly these events, there have been reports associated with very low-volume injections as well.

It is generally assumed that the use of blunt-tipped cannulas, low-volume injections, and low plumber pressure, as most injectors are doing nowadays, reduces vascular risks significantly. ${ }^{22}$ While injection with blunt-tipped cannulas will reduce the risk, it can still occur. Using an airbrush technique, with multiple passes and injecting in multiple layers, restricting to small aliquots of fat injected on each pass, is probably the best we can do to avoid vascular incidents. ${ }^{28}$ The glabella region, nasal dorsum, and nasolabial folds should be approached with particular caution. ${ }^{4}$

\section{Overfilling}

As we discussed in the introduction of this paper, the potential of overfilling the face is perhaps a prime reason why some surgeons prefer not to add fat injections to their arma- mentarium. Indeed, overfilling is an intrinsic risk of fat augmentation. It can occur due to several reasons. Overcorrecting to compensate for anticipated resorption is one. Yet overcorrecting by "design philosophy" is an issue to consider.

While the Ogee curve is commonly advocated as a sign of youth, dogmatically adhering to it can lead to a volume map that lacks esthetic attractiveness. The youthful Ogee curve is the result of a full midface and lateral cheek, subtly flowing into the buccal hollows. While modest filling of the mid cheek can be attractive, in higher volumes it will commonly lead to the kind of apple-cheeks that are recognized as unnatural. $^{36}$ The sudden and deeper transition into the aged buccal hollows aggravates this effect, especially in thin patients. Hence our strategy is to gently fill the mid cheek and explicitly transition into the buccal hollows. The same is true for the temporal fossa. It invariably hollows, especially in low BMI cases, and adding volume can rejuvenate. Yet overfilling the hollows, would likewise lead to an unnatural and larger facial structure.

A related error is to focus too much on shadows or hollows, which can lead to an "obliteration strategy." Some shadows are present even at a very young age, yet somehow become disturbing as we age (-Figs. 8).

As - Fig. 8A illustrates, even at the peak of youth, shadows are natural and rather nondisturbing. There is a clear shadow that demarcates the inferior orbit from the mid cheek. Likewise, nasolabial shadows are visible, as is the onset of the labiomental lines. Yet they are all soft and pleasing. Finally, as mentioned before, the transition of the midface peak to the buccal region, is defined by a subtle shadow, giving rise to the so-called Ogee curve. Yet in aging, these same characteristics somehow can have a disturbing effect. Even though the orbital hollow may be slightly deeper, and the lid-cheek junction elongated, it is by a moderate degree mostly, something that Lam also noticed. ${ }^{37}$
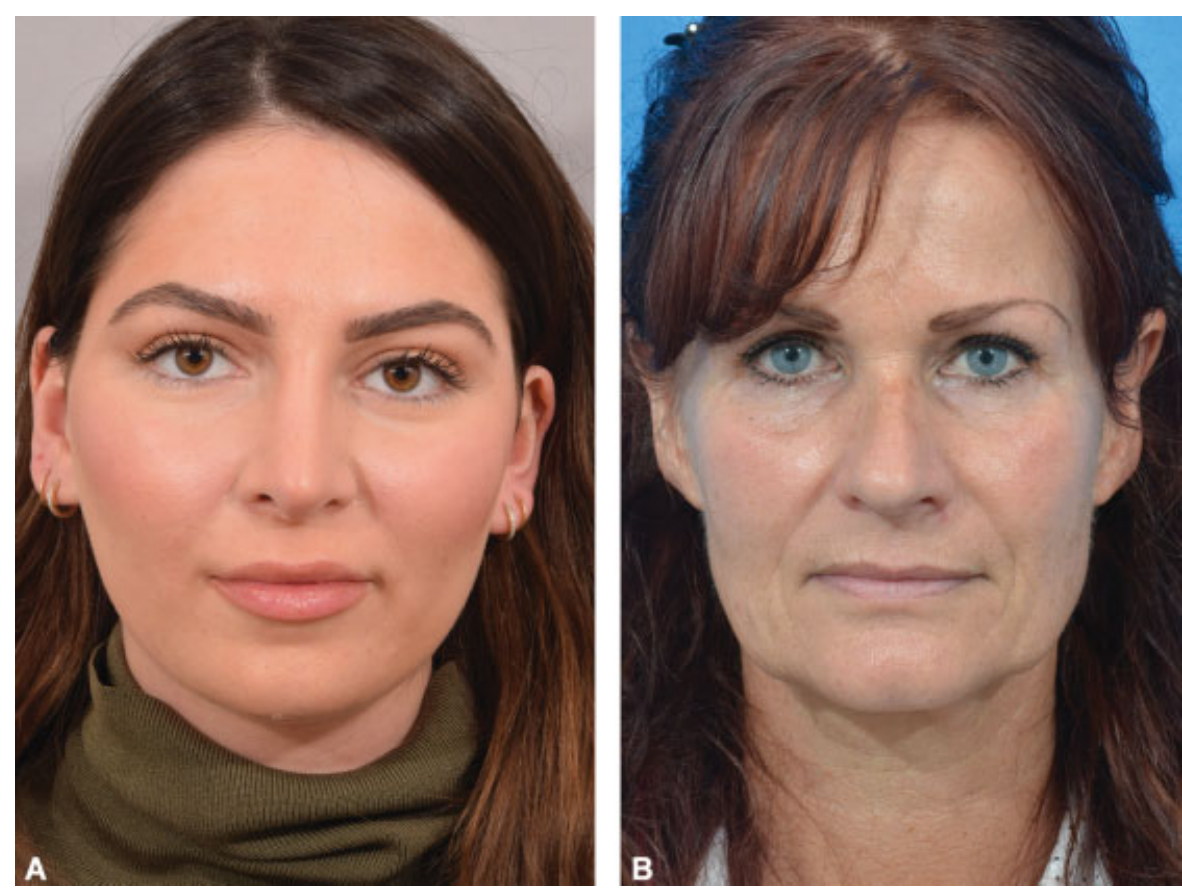

Fig. 8 (A,B) Normal facial shadows in a 25-year-old (left) and 56-year-old (right) woman. 
The same is true for the nasolabial fold and labiomental lines, which are only slightly more prominent as we age. It hence often seems to be more the interplay of multiple smaller changes that we appreciate as an aged look, rather than the lack of shadows per se. Hence simply "obliterating" these shadows is certainly not replicating the natural youthful face, and is rather a stigma of plastic surgery. A relatively large proportion of the population does not aspire this look. ${ }^{7}$

\section{Fat or Surgery?}

Rejuvenating the face as natural, durable, and safe as we can, is what all facial plastic surgeons aspire. Yet there can be different approaches to attain that goal. ${ }^{20}$ CORE facelift techniques have evolved into the golden standard in facelift surgery, as they promise to reposition the sagged tissues, the superficial medial cheek fat pad, and the deep medial cheek fat pad (DMCF) perhaps most importantly, into a more youthful position, in addition to improving the jawline. ${ }^{38-40}$ This standard is advocated both "bottom-up" as well as "topdown."

The bottom-up approach is rather institutionalized in medical literature. The general idea is that deeply understanding the pathophysiology can guide us to techniques that have a high degree of efficacy. Kahn, for example, states that "the best way to develop solutions to a problem is to understand the changes that result in that problem." ${ }^{12}$ Yet while this is undoubtedly true, it can also lead to the kind of theoretical solutions that are unfit for patient care, as we have seen throughout medical history. ${ }^{41}$ It can also lead us to overlook less complex pragmatic solutions. Indeed there are clear biomechanical advantages to CORE facelift surgery. The aged medial cheek fat pads are clearly ptotic and located medio-inferior of the retaining ligaments, which makes permanent repositioning through less extensive surgical techniques vulnerable to relapse. As can be said simply said, the ligaments are in the way. ${ }^{38}$

A top-down perspective is pragmatic and is not much concerned with the underlying pathways, but rather focuses on outcome. Though related of course, the two do not by definition overlap perfectly. From a top-down perspective, some of our leading surgeons have pragmatically and convincingly demonstrated superior results with CORE facelift techniques. ${ }^{22,38,39}$ Some debate remains however, as to how (in which plane) to safely and most effectively release and mobilize the midface fat pads, that partially lie anterior to the zygomatic major muscle. $^{42}$

The discursive dialogue between "pragmatists" and "foundationalists" so to say, is one of the oldest in medicine, and is unlikely to be resolved, certainly not by this article. Yet it is critical to acknowledge that to actually tap into the top range of results in the midface within acceptable margins of safety, CORE facelift techniques likely require broad experience, a scope which may actually be beyond the career potential of many facelift surgeons. Chasing a complex goal, as CORE techniques invariably are, in a relatively low volume environment, is likely significantly restricted, bar the absolute most talented of surgeons, which we just cannot assume we are. It is not just about releasing "some" ligaments that makes this maneuver complex, but rather the extent to which one releases the mid cheek. It is the authors belief that the extent of release of these ligaments is the determining factor in achieving great versus awesome results. Moreover, regarding the biomechanical premises, this paradigm assumes that aging is primarily due to decent and redundancy of soft tissues due to loss of support of retaining ligaments, and that actual atrophy of fat pads and the bony scaffold play an insignificant role. While this may be true for some individuals, it is probably not for many others. ${ }^{12}$

Hence volume augmentation has definite benefits, not as a stand-alone procedure per se but certainly as an adjunct to traditional facelift surgery. Particularly the DMCF pads are assumed to be deflated and are the prime targets of fat augmentation. ${ }^{43}$ Fat augmentation can produce results that are superior to face-lifting alone, if surgical experience is accepted as an individual constant. ${ }^{20}$ Finally, fat itself may add a rejuvenating or regenerative impact on skin quality, probably through the presence of the so-called ASCs, which is left unexploited in nonfat techniques. ${ }^{18,44}$ There are hence quite some arguments to consider fat as an adjunct treatment in facelift surgery, especially in those with weaker skull structure and more than average fat atrophy. Fat augmentation can assist to achieve great facelift results, regardless of experience level and technique used.

Conflict of Interest

None declared.

\section{References}

1 Neuber F. Fettransplantation. Chir Kongr Verhandl Deutsche Gesellsch Chir 1893;22:66

2 Lexer E. Freie fettransplantation. Dtsch Med Wochenschr 2010; 36:640

3 Hollander E. Die Kosmetische Chirurgie. In: Joseph M, ed. Handbuch der Kosmetik. Leipzig: Verlag von Velt; 1912:690-691

4 James IB, Coleman SR, Rubin JP. Fat, Stem cells, and platelet-rich plasma. Clin Plast Surg 2016;43(03):473-488

5 Coleman SR. Structural fat grafting. Aesthet Surg J 1998;18(05): 386-388, 388

6 Little JW. Applications of the classic dermal fat graft in primary and secondary facial rejuvenation. Plast Reconstr Surg 2002;109 (02):788-804

7 Lam SM. Fat grafting for facial contouring. Facial Plast Surg 2019; 35(03):278-285

8 Rohrich RJ, Pessa JE. Discussion: aging of the facial skeleton: aesthetic implications and rejuvenation strategies. Plast Reconstr Surg 2011;127(01):384-385

9 Pessa JE, Chen Y. Curve analysis of the aging orbital aperture. Plast Reconstr Surg 2002;109:751-755

10 Kahn DM, Shaw RB Jr. Aging of the bony orbit: a three-dimensional computed tomographic study. Aesthet Surg J 2008;28(03): 258-264

11 Shaw RB Jr, Katzel EB, Koltz PF, et al. Aging of the facial skeleton: aesthetic implications and rejuvenation strategies. Plast Reconstr Surg 2011;127(01):374-383

12 Kahn DM, Shaw RB. Overview of current thoughts on facial volume and aging. Facial Plast Surg 2010;26(05):350-355

13 Pessa JE, Zadoo VP, Mutimer KL, et al. Relative maxillary retrusion as a natural consequence of aging: combining skeletal and softtissue changes into an integrated model of midfacial aging. Plast Reconstr Surg 1998;102(01):205-212 
14 Pessa JE. An algorithm of facial aging: verification of Labros's theory by three-dimensional stereolithography, with reference to the pathogenesis of midfacial aging, scleral show, and the lateral suborbital trough deformity. Plast Reconstruct Surg 2000;106:479-488

15 Pecora NG, Baccetti T, McNamara JA. The aging craniofacial complex: a longitudinal cephalometric study from late adolescence to adulthood. Am J Orthod Dentofacial Orthop 2008;134 (04):496-505

16 Coleman SR, Katzel EB. Fat grafting for facial filling and regeneration. Clin Plast Surg 2015;42(03):289-300, vii

17 Coleman SR. Facial augmentation with structural fat grafting. Clin Plast Surg 2006;33(04):567-577

18 Zuk PA. Stem cell research has only just begun. Science 2001; 293:211-212

19 Moseley TA, Zhu M, Hedrick MH. Adipose-derived stem and progenitor cells as filelrs in plastic and reconstructive surgery. PRS 2006;118(Suppl 3):121-128

20 Marten T, Elyassnia D. Facial fat grafting: why, where, how and how much. Aesthetic Plast Surg 2018;42(05):1278-1297

21 Allen RJ, Heitland AS. Autogeneous augmentation mammaplasty with microsurgical tissue transfer. PRS 2003;112(01):91-100

22 Marten T. Lamellar high SMAS face and mid-face lift: a comprehensive technique for natural appearing rejuvenation of the face. San Francisco

23 Peer L. Loss of weight and volume in human fat grafts. PRS 1950;5 (03):217-230

24 Glasgold RA, Glasgold MJ, Lam SM. Complications following fat transfer. Oral Maxillofac Surg Clin North Am 2009;21(01):53-58, vi

25 Coleman SR. Lower lid deformity secondary to autogenous fat transfer: a cautionary tale. Aesthetic Plast Surg 2008;32(03):4150-4157

26 Maamari RN, Massry GG, Holds JB. Complications associated with fat grafting to the lower eyelid. Facial Plast Surg Clin North Am 2019;27(04):435-441

27 Yoo DB, Azizzadeh B, Massry GG. Injectable 5-FU with or without added steroid in periorbital skin grafting: initial observations. Ophthal Plast Reconstr Surg 2015;31(02):122-126

28 Yoshimura K, Coleman SR. Complications of fat grafting: how they occur and how to find, avoid and treat them. Clin Plast Surg 2015; 42(03):383-388, ix

29 Mineda K, Kuno S, Kato $\mathrm{H}$, et al. Chronic inflammation and progressive calcification as a result of fat necrosis: the worst outcome in fat grafting. Plast Reconstr Surg 2014;133(05): 1064-1072

30 Coleman SR. Structural Fat Grafting. New York, NY: Thieme Medical; 2004

31 Chen B, Song H, Xu M, et al. Treatment of Mycobacterium abscessus infection associated with fat grafting. Surg Infect (Larchmt) 2020; 21(07):639-644

32 Dobke M, Bailey J, Bhavsar D, Saba S, Mailey B. Necrotizing metachronous facial ulcerations after "stem cell face lift". Ann Plast Surg 2013;70(04):392-396

33 Teimourian B. Blindness following fat injection. Plast Reconstr Surg 1988;82:361

34 Beleznay K, Carruthers JD, Humphrey S, Jones D. Avoiding and treating blindness from fillers: a review of World literature. Dermatol Surg 2015;41(10):1097-1117

35 Carruthers A, Carruthers J, Humphrey S. Injecting soft-tissue fillers: overview of clinical use. (C2016 UpToDate

36 Yaremchuk MJ. Making concave faces convex. Aesthetic Plast Surg 2005;29(03):141-147, discussion 148

37 Lam SM. Fat grafting: an alternative or adjunct to facelift surgery? Facial Plast Surg Clin N Am 2013:253-264

38 Jacono AA, Parikh SS. The minimal access deep plane extended vertical facelift. Aesthet Surg J 2011;31(08):874-890

39 Gordon NA, Sawan TG. Deep-plane approach to the vertical platysma advancement: technical modifications and nuances over 25 years. Facial Plast Surg 2020;36(04):358-375

40 Sykes J, Dilger A, Cotofana S. Demystifying the deep plane facelift: what it means anatomically and why it works. Facial Plast Surg 2020;36(04):351-357

41 Baker DC. Commentary on: short scar face lift. Aesthet Surg J 2008;28(02):209-210

42 Cakmak O, Özücer B, Aktekin M, Özkurt FE, Al-Salman R, Emre IE. Modified composite-flap facelift combined with finger-assisted malar elevation (FAME): a cadaver study. Aesthet Surg J 2018;38 (12):1269-1279

43 Pilsl U, Rosmarin W, Anderhuber F. The premaxillary space: a location for filler injection? Dermatol Surg 2014;40(03):301-304

44 Rigotti G, Marchi A, Galiè M, et al. Clinical treatment of radiotherapy tissue damage by lipoaspirate transplant: a healing process mediated by adipose-derived adult stem cells. Plast Reconstr Surg 2007;119(05):1409-1422, discussion 1423-1424 